STP 1615 On Whole Building Air Leakage

\title{
Detection of Air Leakage in Building Envelopes Using Ultrasound Technology
}

Benedikt Kölsch, ${ }^{1}$ Arne Tiddens, ${ }^{1}$ Jacob Estevam Schmiedt, ${ }^{2}$ Björn Schiricke, ${ }^{2}$ and Bernhard Hoffschmidt $^{2}$

${ }^{1}$ Institute of Solar Research, German Aerospace Center (DLR), Karl-Heinz-Beckurts-Str. 13, Jülich, 52428, Germany B. K. http://orcid.org/0000-0003-3564-9822, A. T. http://orcid.org/0000-0003-3714-3008

${ }^{2}$ Institute of Solar Research, German Aerospace Center (DLR), Linder Höhe, Cologne, 51147, Germany J. E. S. http://orcid.org/0000-0002-0794-6769 , B. S. http://orcid.org/00000003-0572-2048, B. H.

\begin{abstract}
Heating energy represents a significant proportion of the total energy consumption of the Federal Republic of Germany. Thus, a reduction of heating energy demand in buildings is one of the central points of German energy saving and climate goals. Considering that the current rate of new building construction is approximately $1 \%$, the most significant energy saving potential lies in existing buildings. The analysis of existing buildings will thus become more important in the years to come. As a result, the development of new measurement systems to assess the need for renovation is required. In this context, detailed information about heat insulation and airtightness is essential. The current approaches to detect air leakage in building envelopes are mostly time consuming and expensive. Therefore, the development of an advanced and more inexpensive method is a vital step. In this paper, we
\end{abstract}


present a study about the feasibility and suitability of ultrasound technology to evaluate the size of air leakages in building envelopes. The advantage of ultrasound technology is that the corresponding wavelengths are small enough to penetrate small openings and that disturbing background noise is usually low. Moreover, the frequencies are above the human threshold of hearing; hence, working and living inside the building would be affected less by the measurement than by a blower door test or leakage detection with audible sound. To examine the suitability of this technology, we use an experimental setup of ultrasound microphones and a speaker in a test chamber. Within this setup, we measured different hole sizes and assessed whether they could be detected using ultrasonic frequency swept signals.

\section{Keywords}

air leakage, ultrasound, leakage size, acoustics, frequency sweep, building envelope, energy performance, Huang-Hilbert transformation

Copyright (c) 2019 by ASTM International, 100 Barr Harbor Drive, PO Box C700, West Conshohocken, PA 19428-2959. 


\section{INTRODUCTION}

Climate protection and environmental degradation have evolved as prevailing global topics over the past few years. In addition to the energy, industry, and transport sectors, the building sector is responsible for approximately a third of global primary energy consumption. ${ }^{1}$ The German government has committed to reducing greenhouse gas emissions from 1990 to 2020 by $40 \%$, and by up to $55 \%$ by $2030 .{ }^{2}$ Unfortunately, according to the German Federal Environment Ministry, the reduction anticipated until 2015 will reach just $28 \%{ }^{3}$ If Germany misses this next milestone, it could jeopardize all of the following goals. Therefore, an intervention is inevitable.

Uncontrolled airflow through the building envelope contributes significantly to increasing consumption of heating or cooling energy in buildings and thus to higher costs for building owners and to higher production of greenhouse gases in this sector. Aside from this fact, building air leakage can lead to an impairment of indoor air quality in ventilated buildings, ${ }^{4}$ can cause construction damages through interstitial condensation of moisture inside the walls, and can adversely affect the performance of existing ventilation systems. ${ }^{5}$

Typically, building envelope airtightness ${ }^{6,7}$ is ascertained by the fan pressurization method ("blower door test"), which is standardized (e.g., in ASTM E779-19, Standard Test Method for Determining Air Leakage Rate by Fan Pressurization). ${ }^{7}$ Although the blower door test can calculate the airflow rate at pressure differences of $50 \mathrm{~Pa}$ or higher, it does not serve the purpose of determining the airflow under natural conditions. The power law allows the extrapolation from measured data at high pressures to values at low pressures, where natural infiltration in buildings occurs. The measurement is an active intervention, however, and primary functions as an assessment criterion to evaluate air leakage between houses. 
Another method used to identify infiltration is the tracer gas dilution method. This method is described in ASTM E741-11(2017), Standard Test Method for Determining Air Leakage Rate by Fan Pressurization. ${ }^{6}$ A nonreactive tracer gas will be released inside a room and the incoming airflow, which has no tracer (e.g., outdoor air) or has less tracer (e.g., from adjacent spaces) in it, leads to reduced concentration of the tracer gas, which will be monitored over time. The specific requirements related to the measurement devices are demanding in this context. Moreover, while using either of these two measurement methods, it is not possible to utilize the building in the usual manner, as utilization of the building by residents will affect the airflow rate. Although these methods are widely used in the industry, their shortcomings make the development of an advanced and more inexpensive method necessary.

The objective of this work is to examine the suitability and feasibility of ultrasound technology to detect air leakage spots and their size in buildings. The frequency of ultrasound is higher than the human threshold of hearing, and it promises to affect working and living less while measurements are taken than the previously described methods. Additionally, the wavelengths are short enough to penetrate small openings, and the expected background noise is typically low. Compared with the blower door test, an acoustic method does not require a pressure gradient, enclosed spaces, and a priori work on sealing vents.

Investigations about using sound to locate infiltration openings in building envelopes were conducted in 1979 . Keast et al. ${ }^{8}$ determined that the sound at cracks is approximately twice as loud compared with sealed locations. ASTM E1186-17, Standard Practices for Air Leakage Site Detection in Building Envelopes and Air Barrier Systems ${ }^{9}$ refers to a technique using sound to detect air leakage sites in building envelopes. This standard recommends the use of a broadband sound source (e.g., white noise) at one side of the wall and a stethoscope or a 
microphone at the other side. The aim is to quantify local increases in sound intensity, which represent potential air leakage sites. Raman et al. ${ }^{10}$ already presented an approach to detect leakage locations in the building envelope using a microphone array and beam-forming

methods. Furthermore, Bader et al. ${ }^{11}$ have explained a method that visualizes the ultrasound intensity to detect leakage spots in building envelopes. Tiddens et al. ${ }^{12}$ patented a system that uses ultrasound to localize leaks in building envelopes. Compared with the previous literature, this paper focuses more on the feasibility of determining leakage sizes.

The presented work is part of ongoing research activities to make remote-sensing techniques applicable for thermal energy analyses of buildings. ${ }^{13}$ The ultimate goal is to develop a toolbox of measurement and analysis methods to determine the thermal properties of building envelopes quickly and accurately. Thus, single buildings or whole districts may be examined in a short time to obtain crucial information for the development of renovation strategies or about loads of the energy networks.

\section{State-of-the-Art Fan Pressurization Method}

Currently, the fan pressurization method (also known as "blower door test") is the most frequently used method for quantifying infiltration in building envelopes. This method is specified in various standards, including DIN EN ISO 9972, Thermal Performance of Buildings $^{14}$ or ASTM E779 ${ }^{7}$ and is used for the following purposes:

1. Measuring the air leakage in building envelopes to meet the requirement of airtightness to fulfill energy performance standards (e.g., passive house)

2. Comparing the relative airtightness of different buildings or parts of buildings 
3. Determining the reduction of air permeability after the implementation of improvements

Before the start of the measurements, all external doors, windows, and intentional vents must be sealed. Afterward, one door that leads to the outside will be replaced temporarily with a door with integrated fan, which allows pressure difference to be applied across the building envelope and therefore the airflow induced by air leakage can be measured.

At least two measurement series have to be taken: one pressurizing and one depressurizing the building. Depending on the specific standard that is used (an example of these measurements is shown in figure 1), the pressure difference is $10 \mathrm{~Pa}$ up to 50 or $60 \mathrm{~Pa}$ between the inside and outside. No less than five measurement points should be recorded for each measurement series. The accuracy of these measurements depends heavily on the weather conditions, whereby the velocity of wind should not be higher than $6 \mathrm{~m} / \mathrm{s}$ and the product of the temperature difference (in K) between the inside and outside and the building height (in m) should not exceed $500 \mathrm{mK}$. 


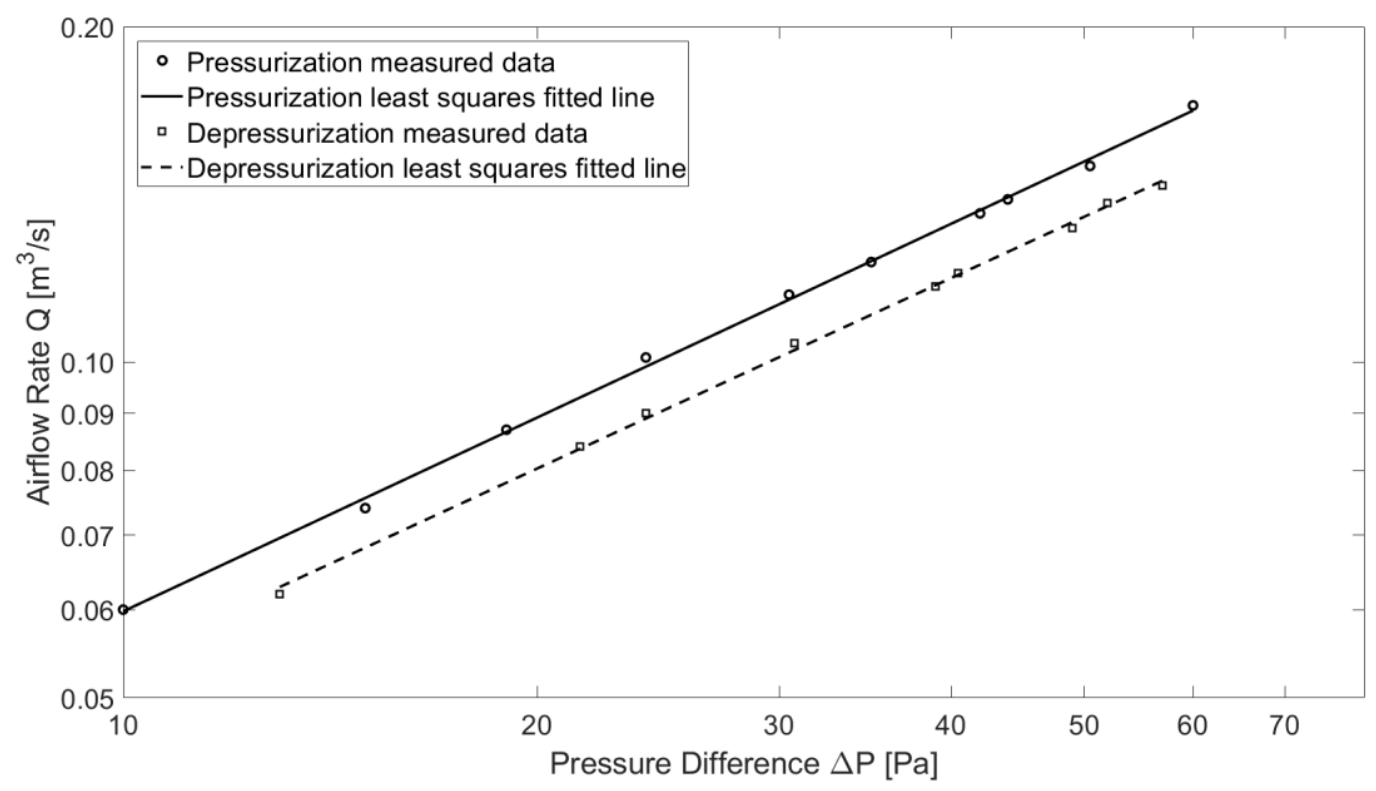

FIG. 1 Example of an air leakage graph with a double logarithmic scale, based on ASTM E779. ${ }^{7}$

Building air leakage is represented by the measured airflow rate $Q$ (in $\mathrm{m}^{3} / \mathrm{s}$ ), which is a function of the pressure difference between the outside and the inside of the building $\triangle P$ (in Pa). This empirical formulation is known as the power law (equation (0)), where $C$ (in $\mathrm{m}^{3} / \mathrm{sPa}^{\mathrm{n}}$ ) represents the flow coefficient, which is a measure of the overall leakage size. ${ }^{15,16}$ The flow exponent $n$ characterizes leakage shape, which is limited to values between 0.5 (turbulent flow) and 1.0 (laminar flow) but typically is in the vicinity of $0.65 .^{17}$

$$
Q=C \Delta P^{n}
$$

The blower door test enables the determination of flow coefficient $C$ and flow exponent $n$, whereas the power law formulation allows the extrapolation to lower pressure differences in cases in which infiltration occurs under natural conditions. The blower door test measures the total air change rate for a whole building, but the meaningfulness of this value is limited. One does not automatically know where exactly and to what extent the leaks exist. Additional 
methods (e.g., smoke tracer or infrared thermography) must be implemented to identify the location of the leaks, which can be time consuming and expensive.

This work shows the potential of using noncontact ultrasound technology to localize leakage spots and to determine their size without applying a pressure difference.

\section{ACOUSTIC FUNDAMENTALS}

Ultrasound is above the human auditory threshold of hearing; hence, working with ultrasound would not disturb inhabitants during measurements. Whereas wavelengths of audible sound in air are in the order of meters, ultrasonic wavelengths reach the order of millimeters, and thus, they are the same size as potential leaks. This paper investigates the coherence between the size of leakage orifices and ultrasonic wavelengths.

An appropriate method to cover a wide range of frequencies at once is the utilization of frequency swept signals, which is implied in the standard DIN EN ISO 18233-SS, Acoustics-Application of new measurement methods in building and room acoustics. ${ }^{18}$ Additionally, the influence of standing waves in an enclosed environment is reduced by the use of frequency sweeps. ${ }^{19}$ The use of a sine sweep, compared with white or pink noise, has the advantage that the amount of energy for each frequency is higher because not all the frequencies are played simultaneously.

\section{Frequency Sweep}

A frequency swept signal changes its frequency with time. The application described in this paper uses a linear frequency sweep function, in which frequency increases linearly in time. ${ }^{20}$ Exponential or logarithmic sweeps are conceivable as well, whereby the frequency function 
changes exponentially or logarithmically over time. In this study, we applied a linear function to ensure that all frequencies are equally distributed over a specified period of time. The formulation for the linear sweep signal $s(t)$, which is valid in the interval of $0 \leq t \leq T$ can be described as follows:.

$$
s(t)=A \cos \left(\theta_{0}+2 \pi f_{1} t+\pi r t^{2}\right),
$$

where:

$A=$ signal amplitude

$\theta_{0}=$ initial phase,

$f_{1}=$ start frequency,

$r=$ sweep rate, and

$T=$ the overall sweep duration.

In this specific implementation $s(t)$ becomes 0 for $t<0$ and $t>T$. Figure 2 shows an example of a linear sweep signal in the time domain over $1 \mathrm{~s}$.

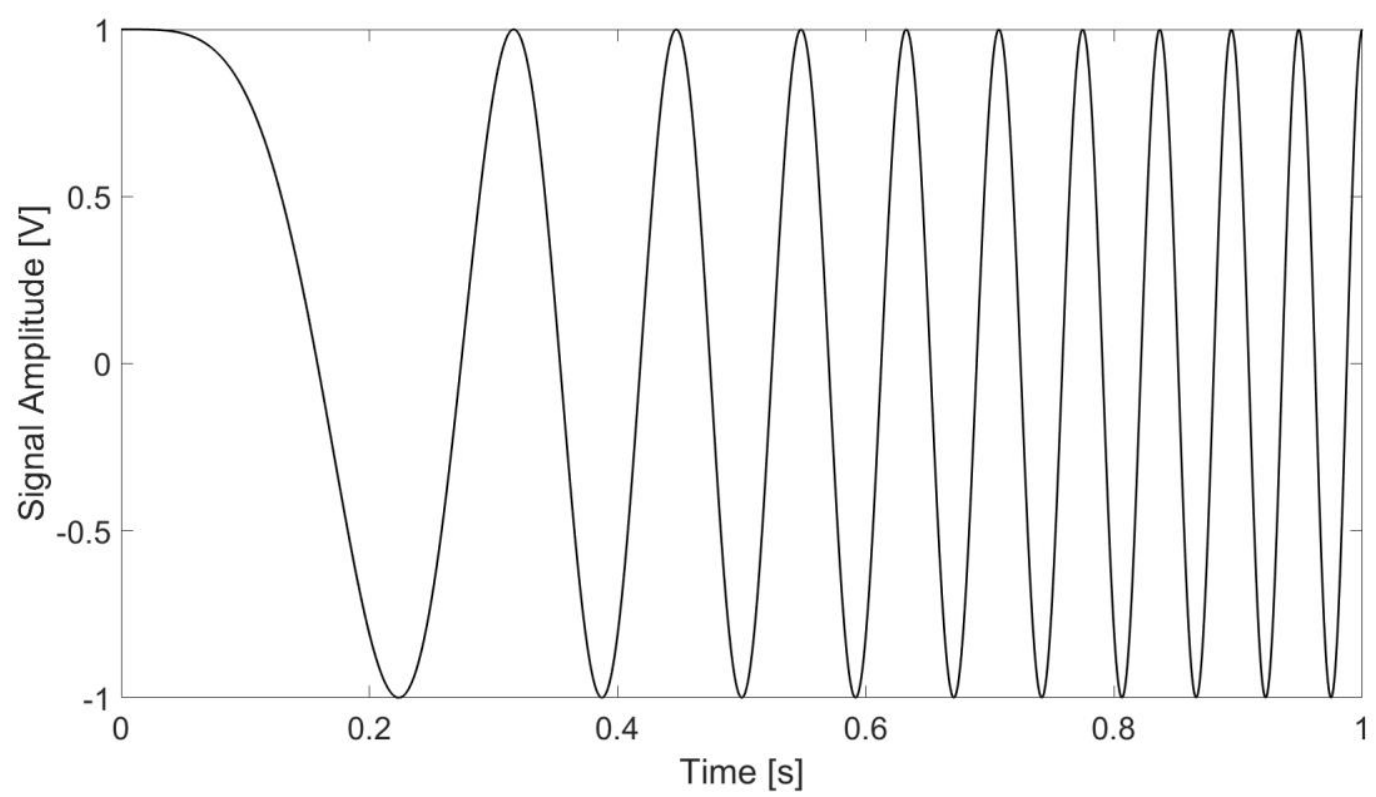


FIG. 2 Example of a linear sweep signal in the time domain.

\section{Fourier Transform}

The Fourier transform shows the frequency content of a signal. Any arbitrary periodic function can be described correctly or at least approximated by a sum of sine and cosine waves. By applying the Euler's formula according to Schenk et al., ${ }^{21}$ the Fourier transform of a continuous signal $s(t)$ can be demonstrated as follows:

$$
X(f)=\int_{-\infty}^{+\infty} x(t)[\cos (2 \pi f t)-i \sin (2 \pi f t)] d t=\int_{-\infty}^{+\infty} x(t) e^{-i 2 \pi f t} d t
$$

The Fourier transform is defined for continuous signals and signals that are unlimited in time. In this case, however, the signal is not continuous. Because of the sampling process, the signal contains a finite number of discrete frequency components; hence, the discrete Fourier transform (DFT) should be applied:

$$
X[k]=\Delta t \sum_{n}^{N-1} x[n]\left[\cos \left(\frac{2 \pi n k}{N}\right)-i \sin \left(\frac{2 \pi n k}{N}\right)\right]=\Delta t \sum_{n}^{N-1} x[n] e^{-\frac{i 2 \pi n k}{N}} .
$$

The DFT requires a long computing time for large data sets; therefore, the fast Fourier transform (FFT), which is a faster algorithm, is used in this work. If the signal length is an exact power of two, the results from FFT are the same as the results from DFT, but with fewer operations and less processing time. The outcome of the calculation is a two-sided spectrum in a complex format.

If a Fourier transform is applied on a linear sweep with constant amplitude and sweep rate, all frequencies between the start and end frequency amplitude equally exist in the frequency spectrum and build a rectangular shape. In comparison, white noise has similar properties in the frequency spectrum. It exhibits a constant distribution of energy in the frequency 
spectrum. $^{22}$ Therefore, a frequency sweep with an infinite bandwidth would have a similar spectral shape. Although white noise is easy to implement, a frequency sweep tends to be the better choice, because of its higher resolution and better signal-to-noise ratio.

Figure 3 illustrates the frequency spectrum of a linear sweep with a start frequency of $20 \mathrm{kHz}$ and an end frequency of $100 \mathrm{kHz}$. In most parts of the sweep spectrum, the spectrum has a rectangular shape except at the beginning and end of the signal, at which point oscillations (called Fresnel ripples) occur. No ripples would occur in a signal with infinite duration, but they appear because of a finite signal bandwidth, which generates discontinuities at the ends of this signal in the frequency spectrum. ${ }^{23}$ These discontinuities should be deleted to ensure that they do not lead to misinterpretation in the evaluation of the measured frequency spectrum.

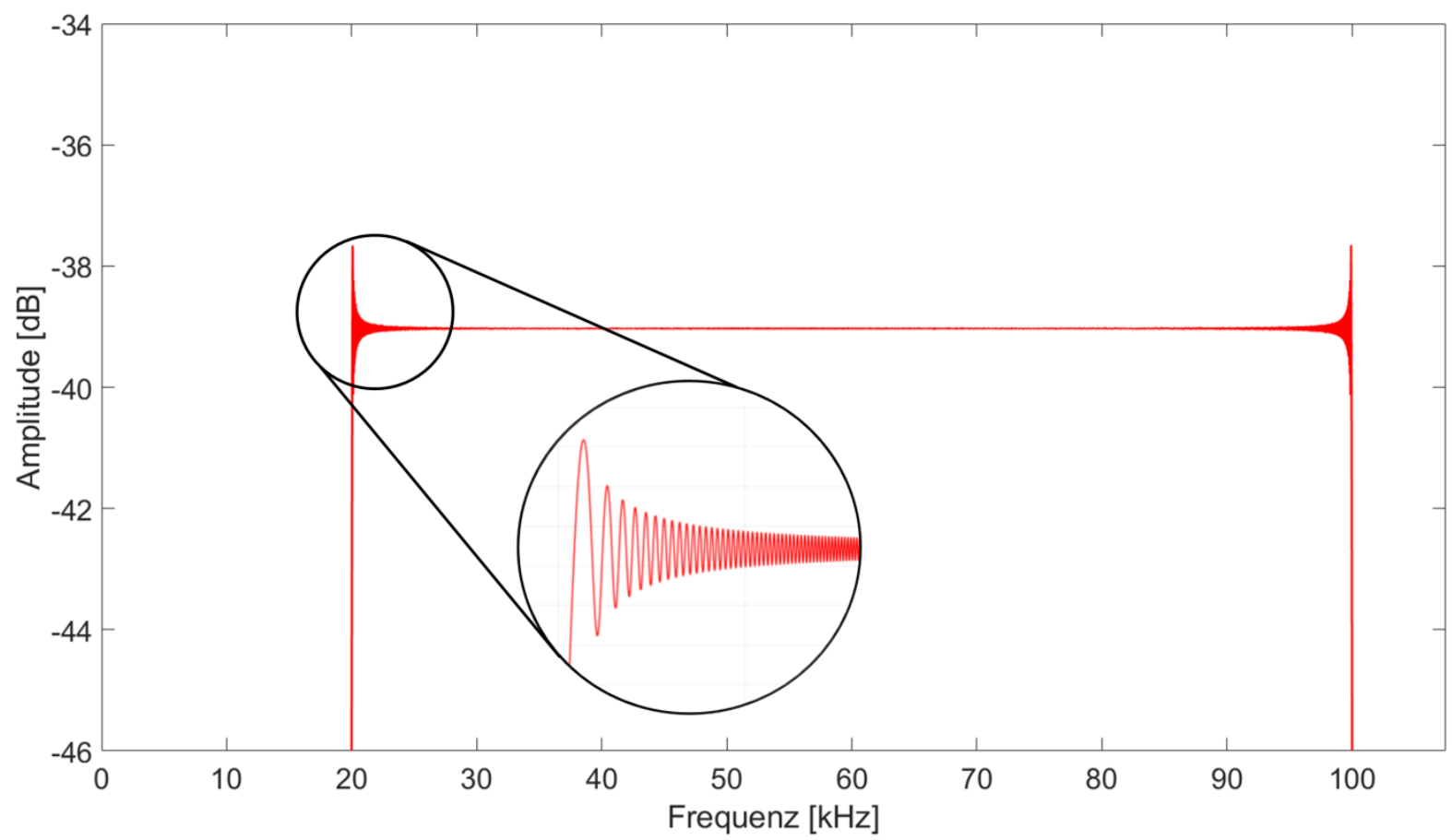

FIG. 3 Frequency spectrum of a linear sweep between $20 \mathrm{kHz}$ and $100 \mathrm{kHz}$. 
According to Aldridge, ${ }^{20}[20]$, the cosine tapered window (or the Tukey window) has been used to taper the signal amplitude at the beginning and the end of the signal:

$$
w_{\text {Tukey }}[n]=\left\{\begin{array}{lr}
\frac{1}{2}\left[1-\cos \left(\frac{\pi n}{N_{1}}\right)\right], & 0 \leq n \leq N_{1} \\
1, & N_{1}<n<N-N_{2} . \\
\frac{1}{2}\left[1-\cos \left(\frac{\pi\left(n-N+N_{2}\right)}{N_{2}}\right)\right], & N-N_{2} \leq n \leq N
\end{array}\right.
$$

The parameters $N_{l}$ and $N_{2}$ express the proportion of the tapered data at the start and end of the sweep signal, whereas the signal remains constant in the interspace. In this work, $N_{1}$ and $N_{2}$ are both chosen to be 0.05 , which promises to remove discontinuities but does not affect the signal bandwidth excessively. For $N_{1 / 2} \leq 0$, the Tukey window segues into a rectangular window, and for $N_{1 / 2} \geq 1$ the window segues into a Hanning window [24]. Figure 4 shows an example of the shape of the resulting signal in the time domain.

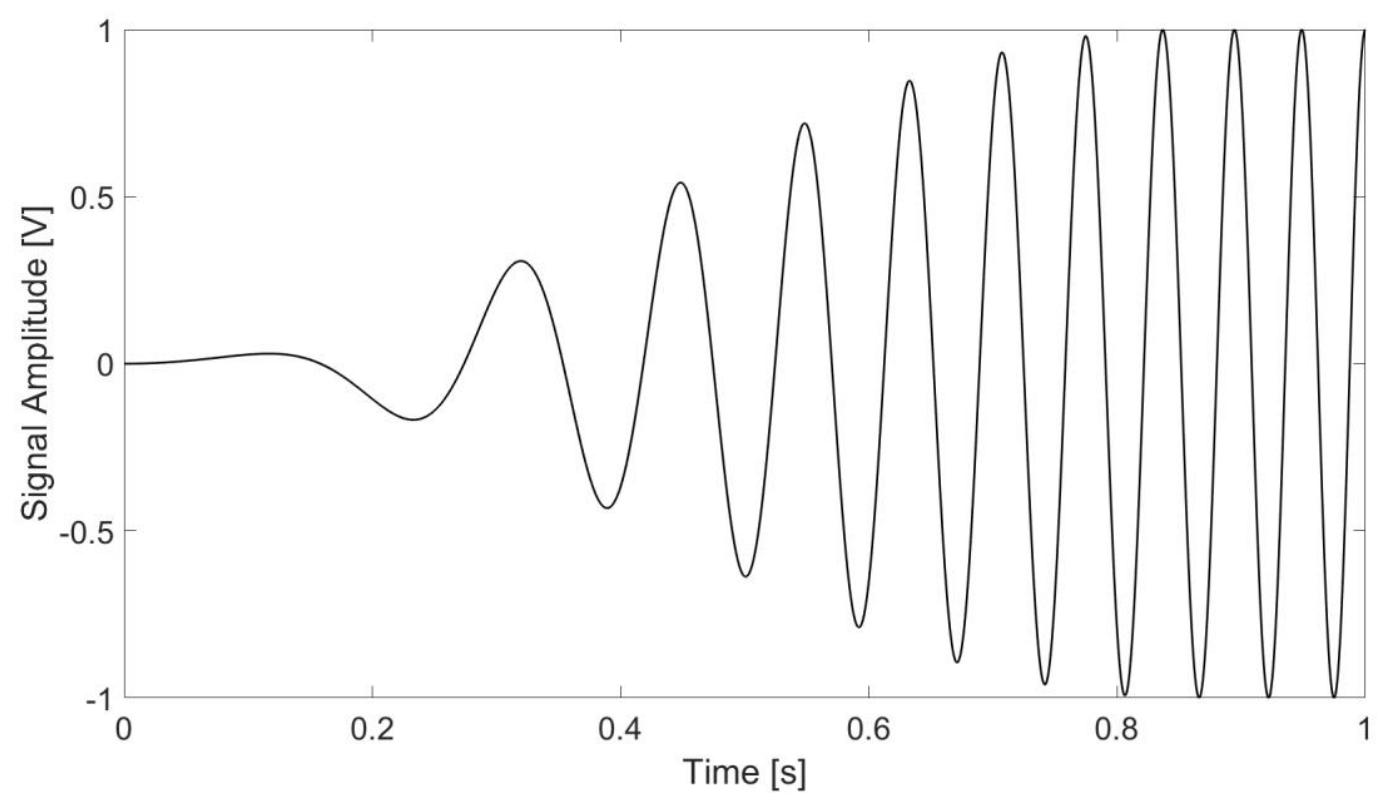

FIG. 4 Example of a linear sweep signal in the time domain with amplitude tapering. 
This method of gradually induced tapering reduces the magnitude of oscillations at both ends of the spectrum, but it results in a slight reduction of spectral bandwidth; in this case, the reduction is approximately $4 \%$. Figure 5 shows the spectrum of the tapered signal with no visible oscillations.

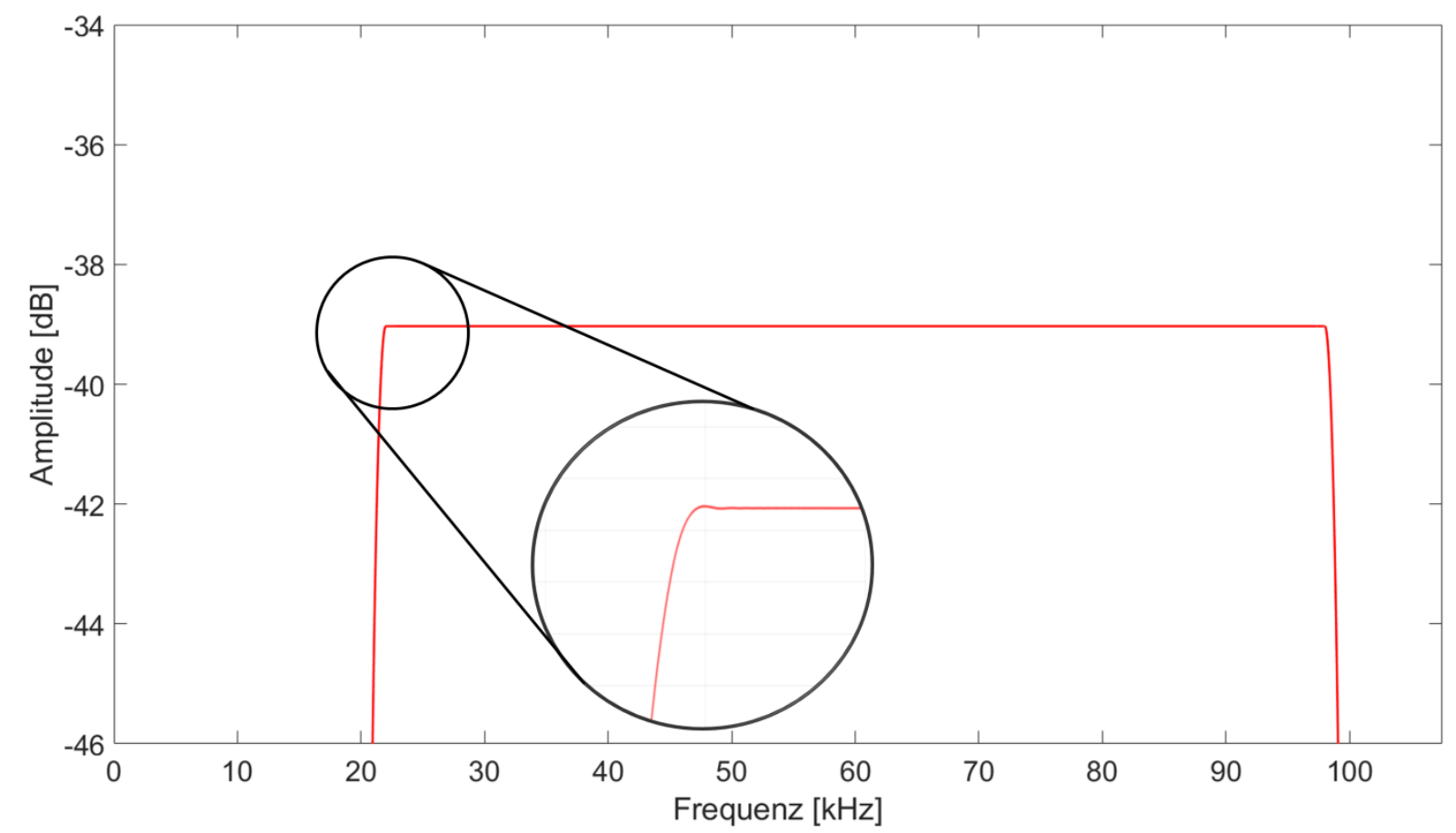

FIG. 5 Frequency spectrum of a linear sweep between $20 \mathrm{kHz}$ and $100 \mathrm{kHz}$ and an applied Tukey window.

\section{HILBERT-HUANG TRANSFORMATION}

In addition to the widely used and previously described Fourier transform, other methods are available to analyze the spectral content of a transient signal. A linear frequency sweep, which we use in this paper, is a typical nonstationary signal. To obtain information about the 
signal behavior at a specific time step for different hole sizes, a high resolution in frequency and time is crucial. Zhou et al. $^{25}$ compared different methods (e.g., short-time Fourier transform, Wigner-Ville distribution, wavelet transform, and Hilbert-Huang transformation $[\mathrm{HHT}])$ to identify the most appropriate time-frequency analysis method. They selected HHT as the most suitable method for analyzing frequency modulated signals.

The short-time Fourier transform can quickly calculate the frequency components of a signal, but it cannot guarantee a high resolution in frequency and time simultaneously. As described in this paper, the Fourier transform decomposes a signal into a sum of sine and cosine functions with constant amplitude and frequency values. Therefore, these parameters remain stationary over the whole signal length regardless of abrupt changes in the original signal. Thus, the outputs of the Fourier transform are averaged over the whole signal length, because we made calculations under the assumption of the stationary ability of the original signal. The HHT enables visualization of sudden changes in the original signal and has a good resolution in time as well as frequency, but it requires more computing time. ${ }^{26}$

\section{Empirical Mode Decomposition}

The empirical mode decomposition (EMD) is a fundamental part of the HHT. ${ }^{27}$ Instead of describing a signal as a sum of sine and cosine functions, the signal is decomposed into intrinsic mode functions (IMF), which are modulated in frequency and amplitude. The original time signal $X(t)$ can then be described as the sum of the IMFs $C_{j}(t)$ and the residual $R(t):$

$$
X(t)=\sum_{j=1}^{n} C_{j}(t)+R(t)
$$


The IMFs should be able to determine the local instantaneous frequency at each position of the signal. An IMF has to fulfill the following requirements to determine meaningful instantaneous frequencies of the signal:

1. The number of zero-crossing and the number of extrema must be equal or should differ not more than one.

2. The mean value of the envelope defined by the maxima and the minima must be zero at any point.

We took three steps to obtain an IMF. First, we identified all maxima and minima of the signal and connected them with two cubic-splines, so that they built the upper and lower envelope of the signal. Second, we calculated the mean value of the upper and lower envelope is calculated for each step and subtracted them from the initial signal to obtain the difference $X_{l}(t)$. Last, the algorithm checked whether the new signal $X_{l}(t)$ corresponded to the previously described requirements of an IMF. If the requirements were not fulfilled, we repeated the first two steps with $X_{l}(t)$. If the new signal met all requirements, we calculated the first IMF $C_{l}$. After that, we obtained a residual by subtracting the IMF from the original signal. We then used this residual signal as a new initial signal, and we repeated all steps of the calculation to find subsequent IMFs. We stopped this process when we reached a certain predefined threshold, or when the residual became nonoscillatory. Here, we selected the stopping criteria according to Rilling et al. ${ }^{28}$ Therefore, the number of IMFs depended on the original signal and was not automatically the same for all signals.

The first IMFs contained higher frequency components and subsequent IMFs contained lower frequency components. The residual function often showed the overall trend of the original signal. 


\section{Hilbert Spectral Analysis}

Each IMF $C_{j}(t)$ was a signal where the Hilbert transform ${ }^{29}$ could be applied:

$$
Z_{j}(t)=C_{j}(t)+j Y_{j}(t)=a_{j}(t) \cdot e^{i \int f_{j}(t) 2 \pi d t}
$$

where:

$Z_{j}(t)=$ analytic signal associated with $C_{j}(t)$.

Both the amplitude $a_{j}(t)$, and the instantaneous frequency $f_{j}(t)$, are a function of time:

$$
\begin{gathered}
a_{j}(t)=\sqrt{X_{j}^{2}(t)+Y_{j}^{2}(t)}, \text { and } \\
f_{j}(t)=\frac{d\left(\arctan \left[\frac{Y_{j}(t)}{X_{j}(t)}\right]\right)}{d t} \cdot \frac{1}{2 \pi} .
\end{gathered}
$$

Finally, the original signal can be reassembled as the sum of the real part $(\mathfrak{R})$ of the Hilbert transform of all individual IMFs:

$$
X(t)=\mathfrak{R}\left[\sum_{j=1}^{\infty} a_{j} \cdot e^{i f_{j} 2 \pi t}\right]
$$

This formulation enabled us to visualize the amplitude and instantaneous frequency as a function of time, which was the Hilbert amplitude spectrum.

\section{Comb-Filtering Effect}

This section explains the comb-filtering effect, which appeared in the conducted measurements. This effect appeared if sound waves with similar frequency and different phase positions were superimposed. In this case, the sound wave generated by the speaker and the sound wave reflected from the front side of the microphone were superimposed, which is shown in figure 6 . Because of the linearity of the sound wave equations, the sound 
pressures of both waves added up, and constructive as well as destructive interference occurred as a function of the relative phase displacement. For instance, a frequency in which the incoming and reflecting waves exhibited a phase displacement of $180^{\circ}$ led to a cancelation of the waves and consequently to a drop in the frequency spectrum. On the contrary, waves with the same phase, intensified each other and led to an increase of the value in the frequency spectrum. ${ }^{19}$

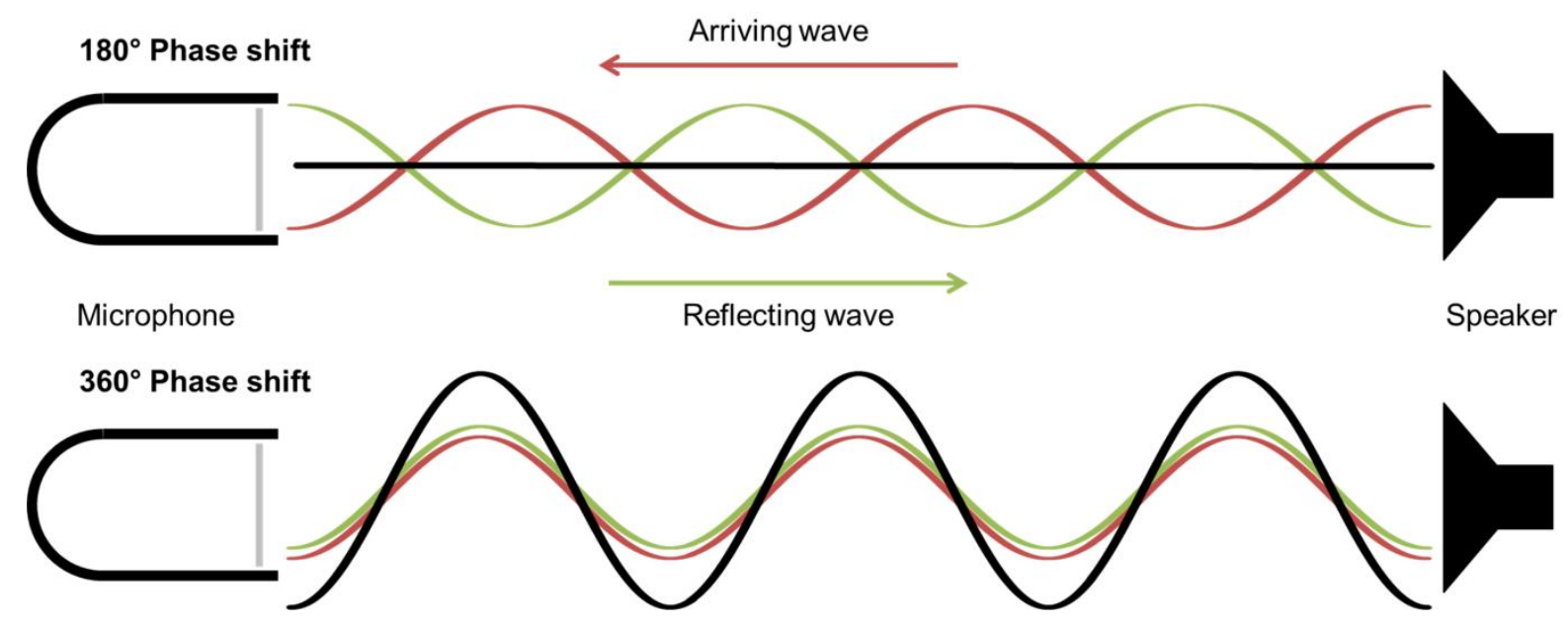

FIG. 6 Comb filter effect.

From the differences between the peaks $\Delta f_{\text {peak }}$ in the frequency spectrum, the distance traveled by the waves can be calculated:

$$
\Delta f_{\text {peak }}=\frac{c}{d}
$$

In this case, $d$ can be presumed as the distance between the speaker and microphone and $c$ as the velocity of sound in air. Therefore, if the comb-filtering effect was visible in the measurement results, additional information about the distance between the measuring devices could be obtained. With increased distance between the microphone and speaker, the 
amplitude of the oscillations in the sound field decreased and the distances between the peaks in the frequency spectrum grew smaller.

\section{EXPERIMENTAL SETUP}

To investigate the effects of leakage spots on swept ultrasonic waves under laboratory conditions, we constructed a test chamber wherein all measurements took place. This acrylic glass test chamber is schematically illustrated in figure 7 . The chamber is divided into two cells (dimensions of each cell: $30.0 \mathrm{~cm} \times 43.5 \mathrm{~cm} \times 31.0 \mathrm{~cm}$ ), which are separated with a replaceable acrylic glass barrier. This barrier contains an orifice, which simulates a leakage with a specific diameter. In this case, we investigated hole diameters from $10 \mathrm{~mm}$ to $4 \mathrm{~mm}$ in $1 \mathrm{~mm}$ steps. We placed a speaker in one cell and a microphone in the other cell, which were both centered in front of the orifice. To suppress ultrasonic vibrations and to ensure that only the signals from the speaker were recorded, both cells were lined with foam. The recorded signal was Fourier transformed to determine the corresponding frequency components. We performed the measurements with a linear upsweep, followed by a downsweep, and a total swept frequency range from 20 to $100 \mathrm{kHz}$. We selected the lower bound of $20 \mathrm{kHz}$, because ultrasound starts at this frequency. In addition, from the upper bound of $100 \mathrm{kHz}$, the damping and absorption of ultrasound in the air becomes too dominant for practical applications. The duration of one sweep was $10 \mathrm{~s}$; hence, it took $20 \mathrm{~s}$ to complete an entire measurement procedure. A signal length that was too short had a nonsatisfactory resolution and signal-to-noise ratio, but a long signal would require too much processing time. Therefore, we selected a total measurement time of 20 s. Between the measurements of various hole diameters, the wall with the orifice was exchanged accordingly. 
The ultrasonic emission source used in this experiment was an ultrasonic dynamic speaker with a frequency range of $1-120 \mathrm{kHz}$. The transducer is a 0.25 -in. condenser microphone with even frequency response and a recommended frequency range of $0.004-100 \mathrm{kHz}$. Both, speaker and microphone remained stationary during measurements.

According to Nyquist-Shannon sampling theorem, which indicates that the sampling frequency should be higher than twice the maximum signal frequency, this measurement setup should have had a minimum sampling frequency of $200 \mathrm{kHz}$ to reach the upper sweep bound of $100 \mathrm{kHz}$. The appearance of higher frequencies than $100 \mathrm{kHz}$ would lead to aliasing effects in the frequency spectrum. Thus, we performed data acquisition using a USB wide dynamic range signal analyzer, which had a sampling frequency of $216 \mathrm{kHz}$ per channel and a resolution of 24 bits. An interface between the data logger and measurement computer, as well as the signal generation and evaluation of the recorded signal, was implemented in MATLAB R2017b ${ }^{30}$.

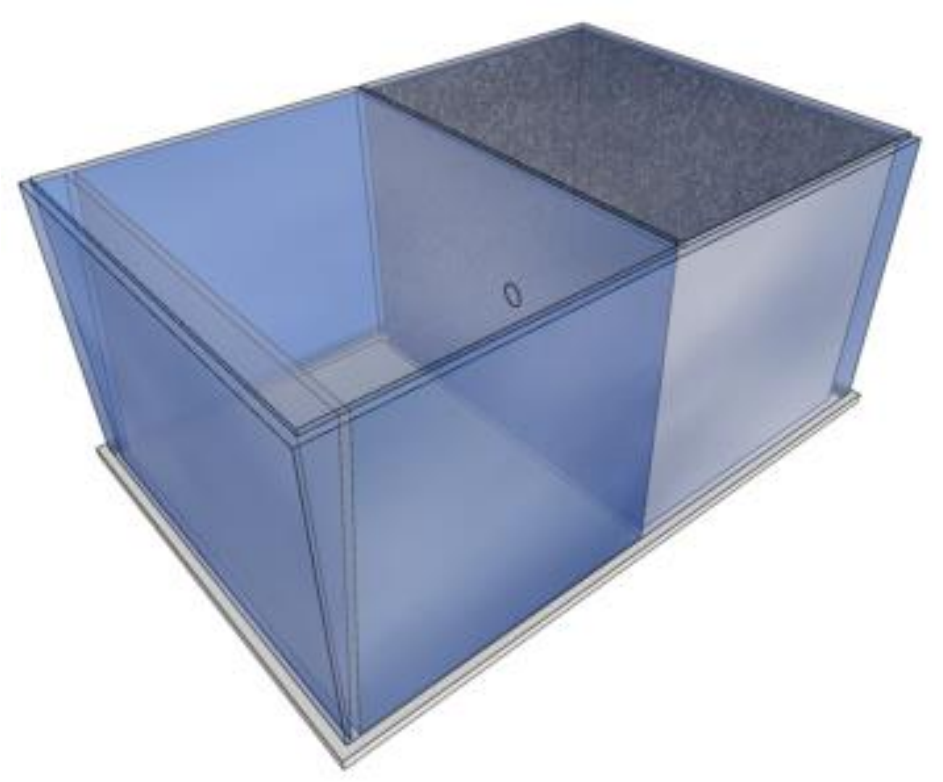

FIG. 7 Test chamber for acoustic measurements. 
A frequency sweep is an exceedingly transient signal. Thus, a high resolution in the frequency spectrum is crucial. To achieve this resolution, we divided the time signal into small FFT-blocks.

With a sampling frequency of $216 \mathrm{kHz}$ and a total duration of the measurement of $20 \mathrm{~s}$, the signal length equaled 4.32 million data points. According to FFT requirements, the block length should be equal to the power of two; therefore, we selected a block length of 8,192 $\left(=2^{13}\right)$. This length was equivalent to less than $2 \%$ of the signal length and was a compromise between an appropriate length of the sequence and computing time. Furthermore, the FFT required a periodical signal to obtain ideal results, but the measurements were not periodic. Therefore, we multiplied the signal time block by a Hanning window function (equation (0)), which reduced the ends of the block gradually to zero and thus resulted in a continuous signal sequence ${ }^{31}$.

$$
w_{\text {Hann }}[n]=\frac{1}{2}\left(1-\cos \left(\frac{2 \pi n}{N}\right)\right) \quad n=1,2, \cdots, N-1 .
$$

The applied window function weighs the signal ends less, however, and the signal information gets lost. As a consequence, we used a moving window with an overlap of $95 \%$ of the previous block in this analysis.

\section{EXPERIMENTAL RESULTS}

The results of the FFT analysis for a linear frequency sweep are shown in figure 8 . The measurement took place inside the previously described test chamber with a leak-proof wall separating the speaker and microphone. In addition to the frequency spectrum, the 
corresponding wavelength is shown in figures $8-10$, for which the velocity of sound in air $c$ was required. The velocity of sound depends nearly linear on the room temperature. According to Bergmann, ${ }^{32} c$ can be approximated as follows:

$$
c=c_{0} \sqrt{1+\beta(T-273.15)} \approx 331.3+0.6(T-273.15)
$$

where $c_{0}$ is equivalent to the velocity of sound at $0^{\circ} \mathrm{C}$ and standard atmospheric pressure, $\beta$ is the thermal expansion coefficient (in $\mathrm{m} /\left(\mathrm{m}^{\circ} \mathrm{C}\right)$ ) of air, and $T$ is the ambient temperature (in $\mathrm{K})$. The equation is valid as long as the wavelength is not in the same order as the free path between the molecules, thus until roughly $10^{8} \mathrm{~Hz}$. Additionally, the effect of dispersion can be neglected within this frequency range. By covering a swept frequency range between 20 and $100 \mathrm{kHz}$, the wavelengths were in a range between 17.3 and $3.5 \mathrm{~mm}$ considering a room temperature of $+23.5^{\circ} \mathrm{C}$.

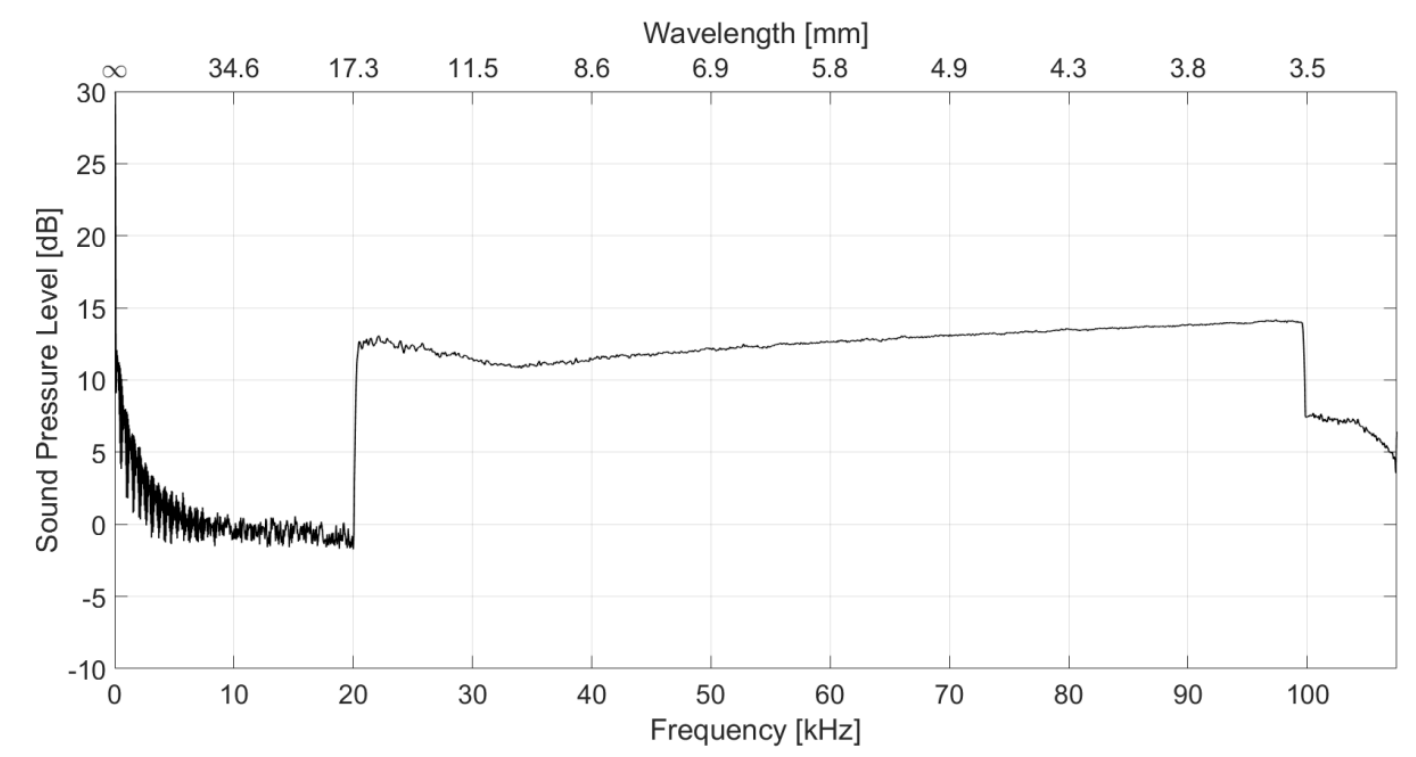

FIG. 8 Frequency spectrum of sweep measurement between 20 and $100 \mathrm{kHz}$ with a separating wall and without a hole in the test chamber. 
An almost rectangular shape in the frequency spectrum is identifiable in the measurement plot (fig. 8). Because of the use of the Tukey window function (equation (0)), the bandwidth was slightly smaller here; the real rectangle in the frequency spectrum started after $20 \mathrm{kHz}$ and finished before $100 \mathrm{kHz}$.

The recorded swept frequency signal ended at approximately $100 \mathrm{kHz}$ but it did not entirely fall back to $0 \mathrm{~dB}$. Although the generated signal was a pure sine wave, the speaker was incapable of producing pure sine waves without harmonic overtones, which were apparent above a frequency of $100 \mathrm{kHz}$. This measurement did not show any effect of comb filtering in the frequency spectrum because the energy of transmitted sound through the wall was too low.

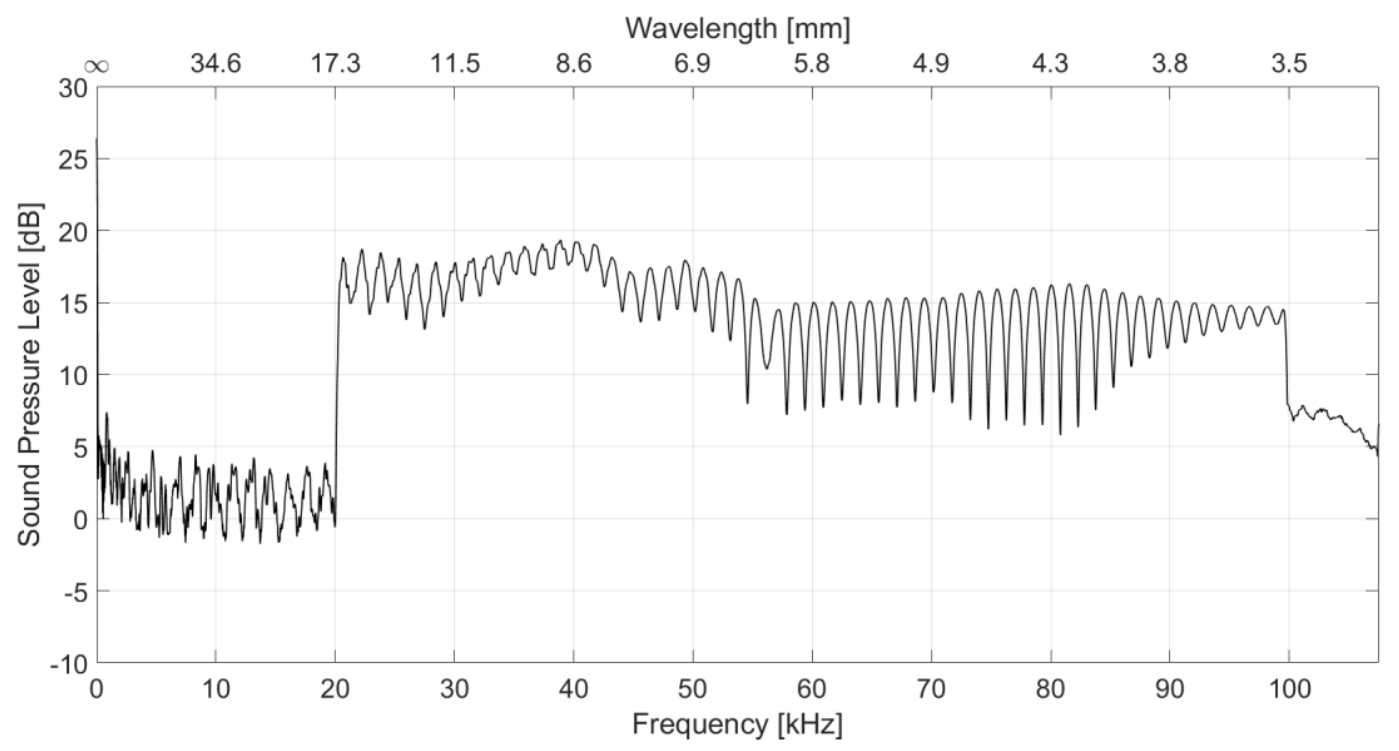

FIG. 9 Frequency spectrum of sweep measurement between 20 and $100 \mathrm{kHz}$ without separating wall in the test chamber. 
In figure 9 the same measurement is taken but without a separating wall inside the test chamber. Here, the previously described comb-filtering effect, which resulted in the amplification and attenuation of certain frequencies occurred in the frequency spectrum, but the rectangular shape of the spectrum was still apparent from 20 to $100 \mathrm{kHz}$. The distance between the frequency peaks $\Delta f_{\text {peak }}$ equaled approximately $1.5 \mathrm{kHz}$, which, according to equation (0), would lead to a distance between the microphone and speaker of roughly $23 \mathrm{~cm}$. This value correlated with the distance between the speaker and microphone tip in the measurement setup. The measurement with a separating wall and without any opening did not show any effect of comb filtering in the frequency spectrum because the energy of transmitted sound through the wall was too low.

In the frequency range between 20 and about $55 \mathrm{kHz}$, the sound pressure level, for the most part, was higher than the measurement with a separating wall. Therefore, the damping effect of the wall appeared to be the greatest in this part of the spectrum. The damping of the sound pressure level was roughly $6 \mathrm{~dB}$ in this range, which equaled a reduction of the sound pressure by half, compared with the measurement shown in figure 8 .

These two measurements function as references for subsequent tests. 

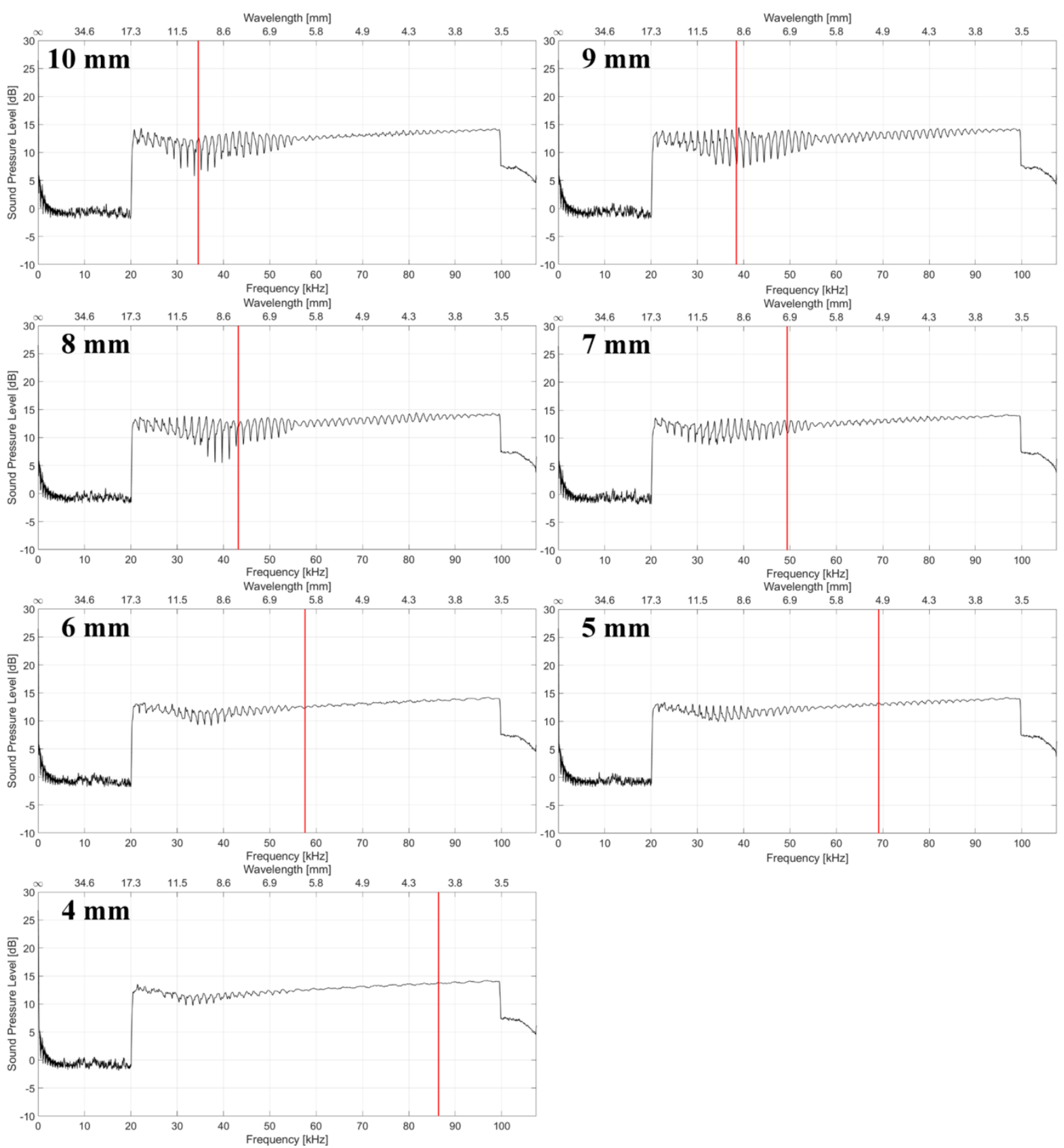

FIG. 10 Frequency spectra of sweep measurements between 20 and $100 \mathrm{kHz}$ with a separating wall and different hole sizes in the test chamber; measurements with hole sizes from $10 \mathrm{~mm}$ to $4 \mathrm{~mm}$ are displayed (vertical lines).

Next, the experiments have been performed with separating walls and different sizes of openings. Figure 10 shows measurements with diameters of the hole inside the separating 
wall from $10 \mathrm{~mm}$ (upper-left part of figure 10) up to $4 \mathrm{~mm}$ (bottom left part) and $1 \mathrm{~mm}$ steps between them. The vertical line indicates the frequency at which the diameter of the hole and the wavelength are theoretically the same.

The comb-filtering effect is most visible for large hole diameters between $10 \mathrm{~mm}$ and $7 \mathrm{~mm}$. Furthermore, with increasing hole diameter, a weak correlation between the range of the maximum oscillation peaks in the frequency spectrum and the location of the hole diameter (vertical lines in figure 10) can be seen. At smaller hole diameters between $6 \mathrm{~mm}$ and $4 \mathrm{~mm}$, oscillations in the lower frequency spectrum are still visible, but considerably smaller. For these smaller hole diameters, the location of the predominant oscillations did not match the location of the hole diameters anymore, but a distinction between these measurements and the measurement of a leak-proof wall in figure 8 remained possible.

We applied the previously described HHT algorithm to measurements with hole diameters of $4,6,8$, and $10 \mathrm{~mm}$ in the separating wall inside the test chamber. To obtain a better time resolution for these measurements, we extended the measurement period to $28 \mathrm{~s}$, and we used a single upsweep $(20-100 \mathrm{kHz})$. The frequency swept signals had the lowest frequency components at the beginning and the highest components at the end of the signal.

Figure 11 shows the results of the EMD for a single frequency sweep and a hole diameter in the separating wall of $10 \mathrm{~mm}$. The first plot shows the original recorded signal and the two corresponding IMFs are plotted below. At IMF1, high-frequency components appeared predominantly, whereas IMF2 primarily showed low-frequency components. At the lowest position, we illustrated the residual function (red line), which contained noise and the overall trend of the signal. The residual function was not used again in the analysis and calculation of the Hilbert amplitude spectrum. 
The Hilbert spectrum in figure 12 was generated from the IMFs used in figure 11 . The Hilbert spectrum shows the magnitude of the instantaneous frequency components over time as an overlay of IMF1 and IMF2 (according to equation (0)). All dark areas represent higher amplitude values of the frequencies. Here, the frequency sweep from low frequency $(20 \mathrm{kHz})$ to high frequency $(100 \mathrm{kHz})$ was visible. Furthermore, because of the comb-filtering effect, the attenuation and amplification of frequencies at specific time steps were apparent throughout the whole measurement period. At approximately second 19, we observed a sudden increase of high-frequency components. This sudden increase is yet to be studied in detail.
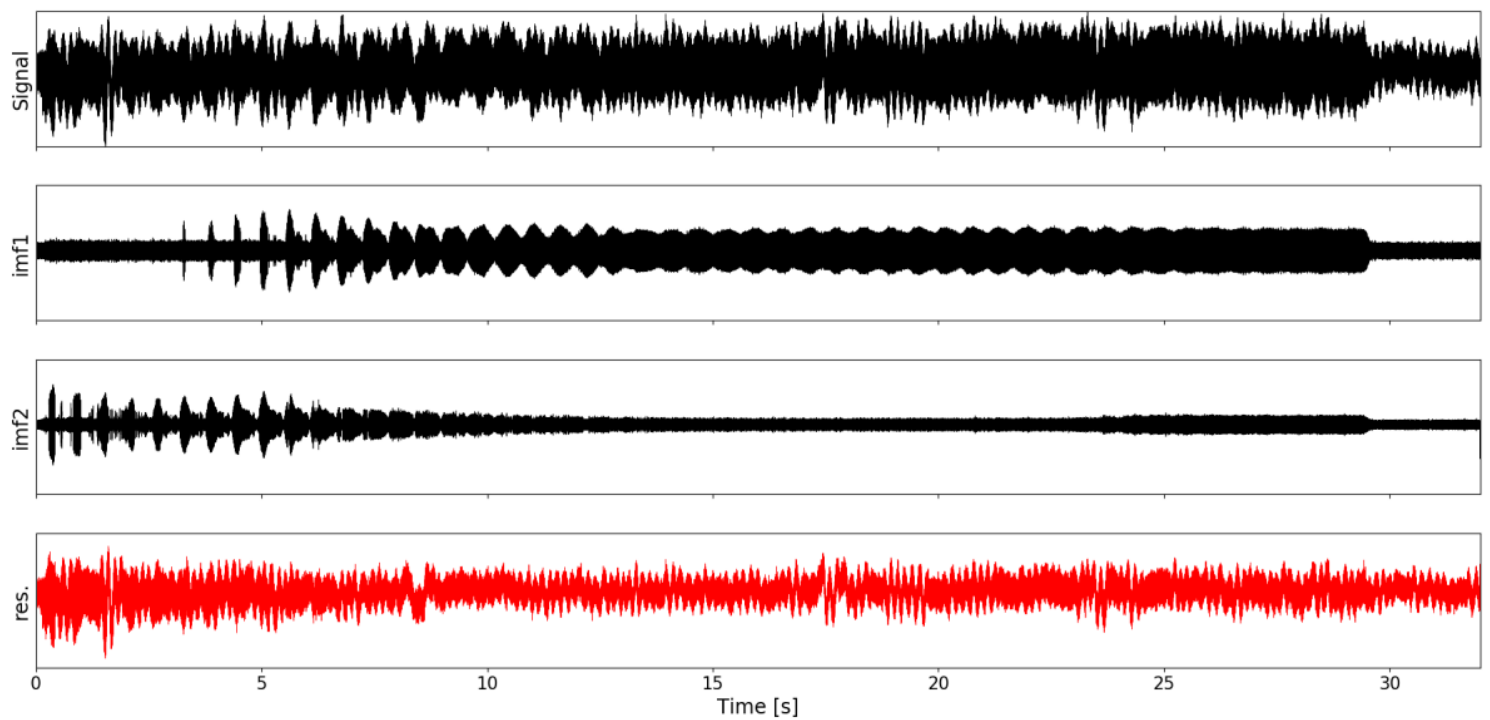

FIG. 11 EMD of the time signal into two IMFs and the remaining residual function (res.) of sweep measurement between 20 and $100 \mathrm{kHz}$ with a separating wall and a hole size of $10 \mathrm{~mm}$. 


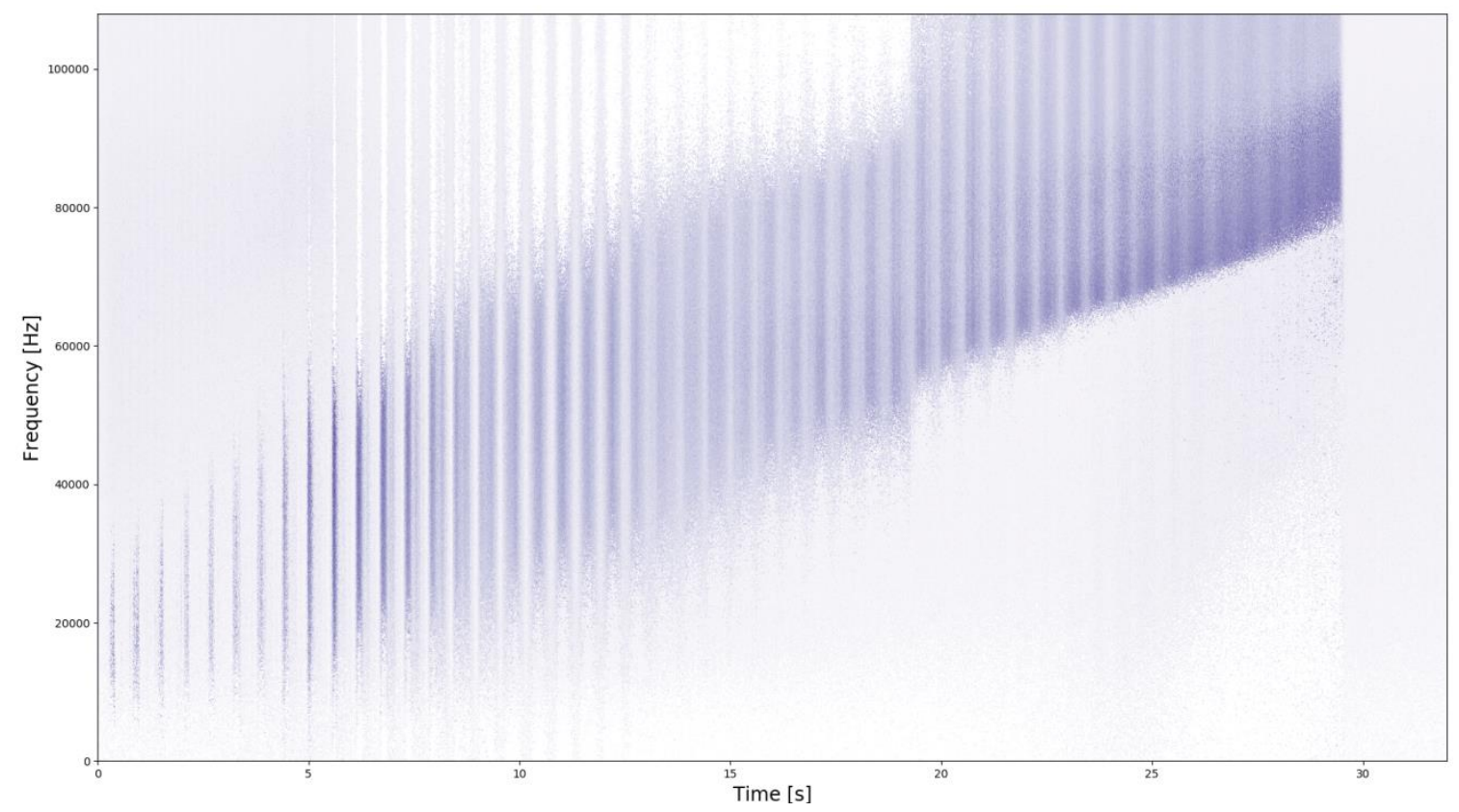

FIG. 12 Hilbert spectrum of sweep measurement between 20 and $100 \mathrm{kHz}$ with a separating wall and a hole size of $10 \mathrm{~mm}$.

Figure 13 presents the EMD of the measured time signal for a hole diameter of $8 \mathrm{~mm}$. Similar to the EMD performed for the measurement for a hole diameter of $10 \mathrm{~mm}$ (fig. 11), we calculated two IMFs. The EMD enabled us to filter unwanted distortions from the original signal, which appeared, in seconds 9 and 14. These distortions are no longer visible in the IMFs and appear only in the residual function.

The shape of the corresponding Hilbert spectrum, which is shown in figure 14, is similar to the Hilbert spectrum in figure 12. In this measurement, the comb-filtering effect was still visible but was slightly mitigated primarily in the first $10 \mathrm{~s}$. 

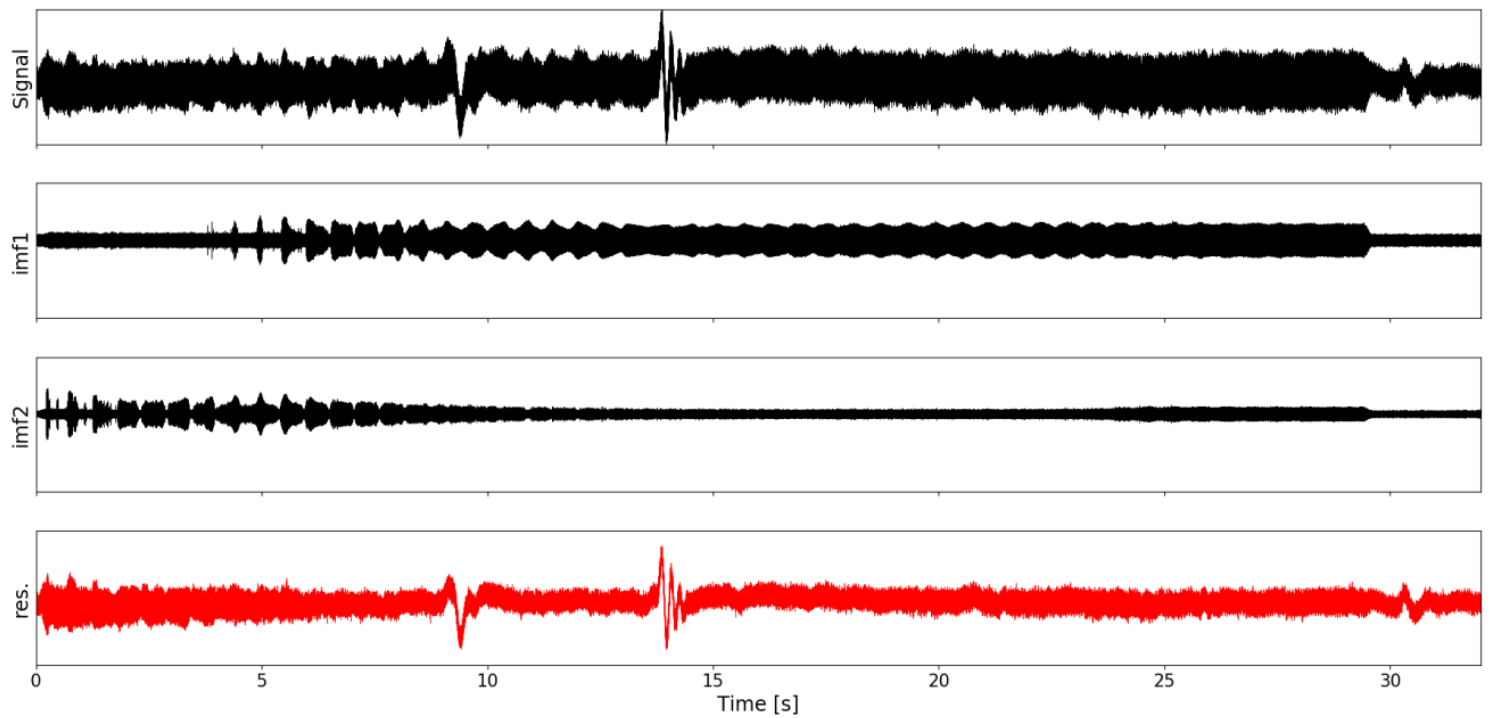

FIG. 13 EMD of the time signal into two IMFs and the remaining residual function (res.) of sweep measurement between 20 and $100 \mathrm{kHz}$ with a separating wall and a hole size of $8 \mathrm{~mm}$.

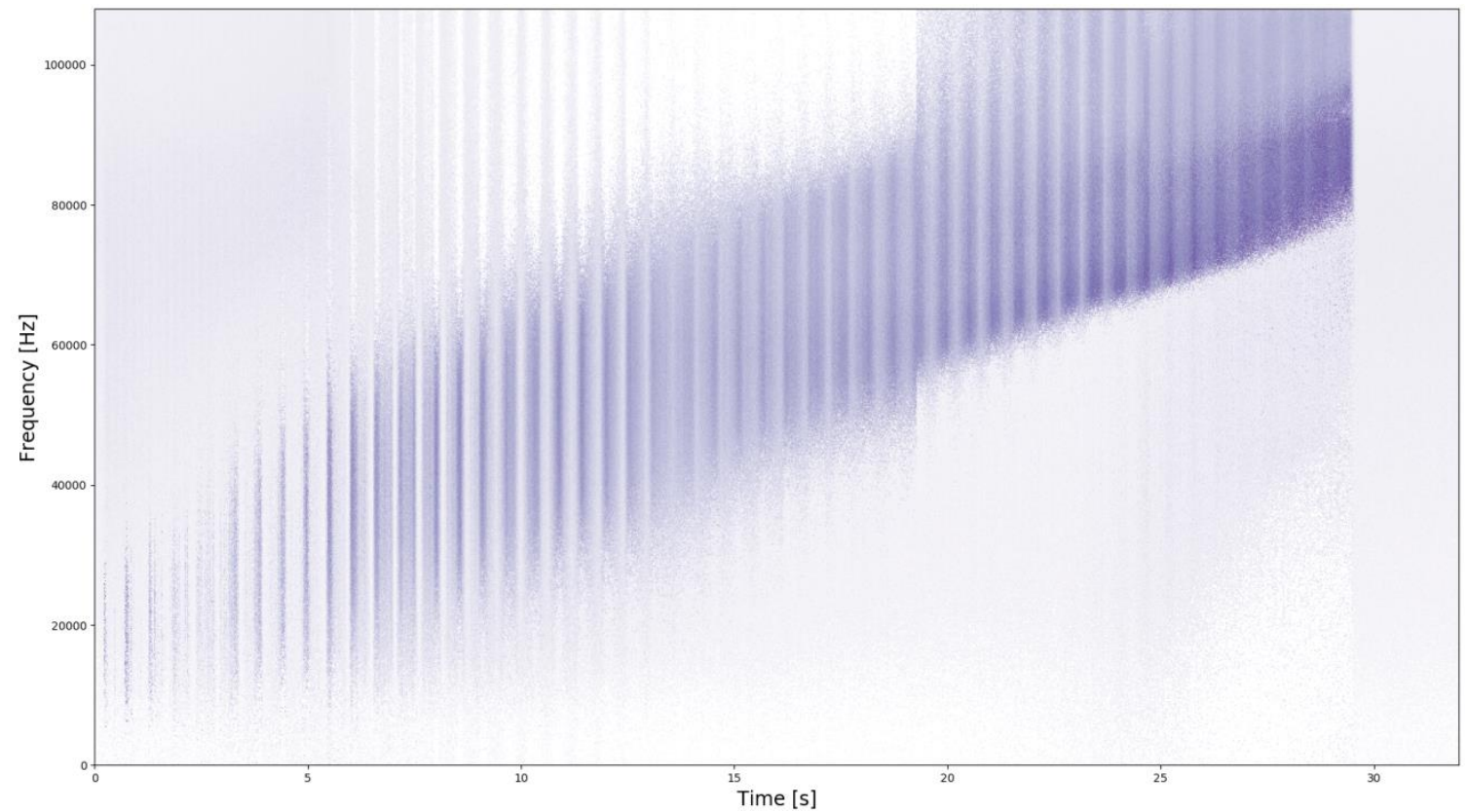

FIG. 14 Hilbert spectrum of sweep measurement between 20 and $100 \mathrm{kHz}$ with a separating wall and a hole size of $8 \mathrm{~mm}$.

The EMD of the signal for a $6 \mathrm{~mm}$ hole (fig. 15) differed from previously shown EMDs concerning the number of calculated EMDs. Here, we calculated four IMFs, in which the first 
IMF represented the high-frequency components between second 6 and 28, whereas the other three IFMs had maxima predominantly in the low-frequency range of the signal, which occurred in the first seconds. The EMD for the measurement of the smallest hole size (4 $\mathrm{mm})$ is shown in figure 17.

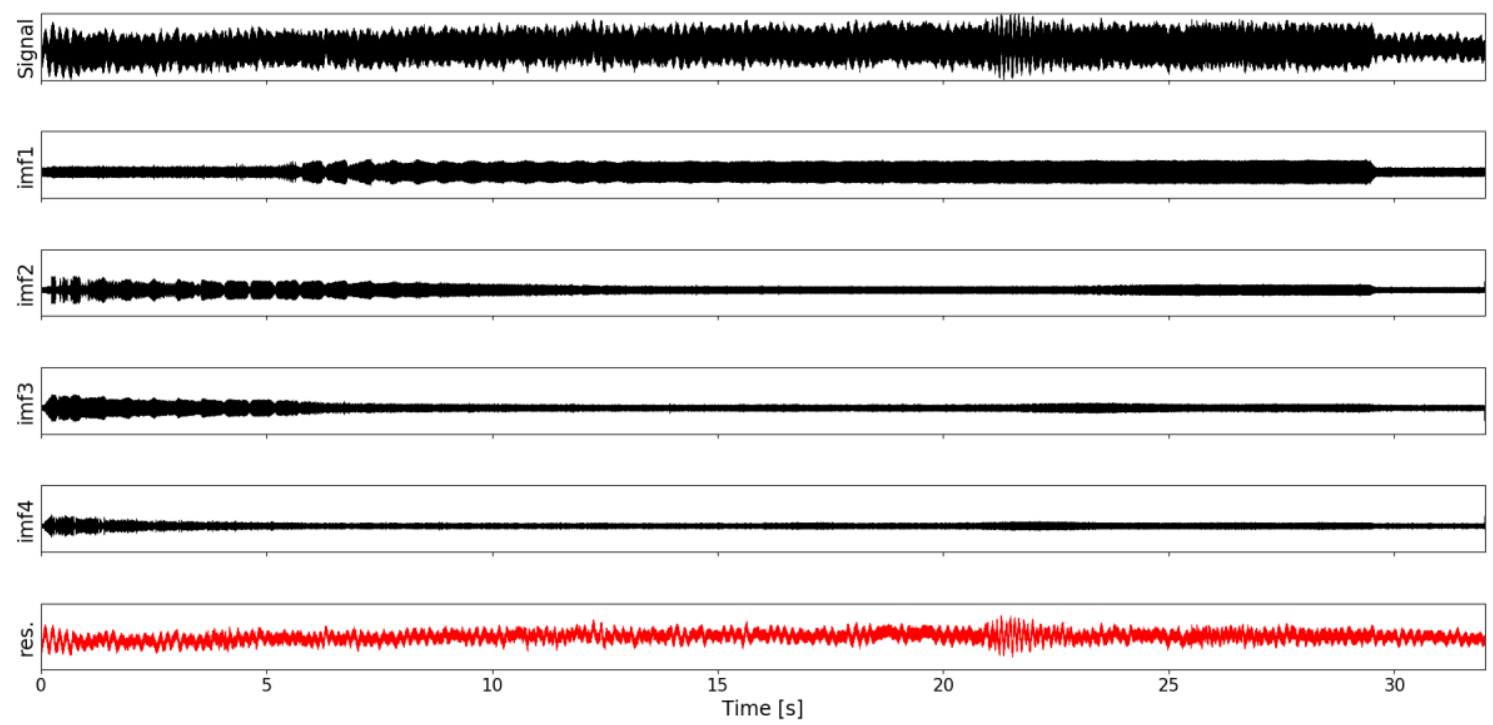

FIG. 15 EMD of the time signal into four IMFs and the remaining residual function (res.) of sweep measurement between 20 and $100 \mathrm{kHz}$ with a separating wall and a hole size of $6 \mathrm{~mm}$.

Figures 16 and 18 visualize the Hilbert spectrum of measurements with hole diameters of 6 and $4 \mathrm{~mm}$ in the separating wall. 


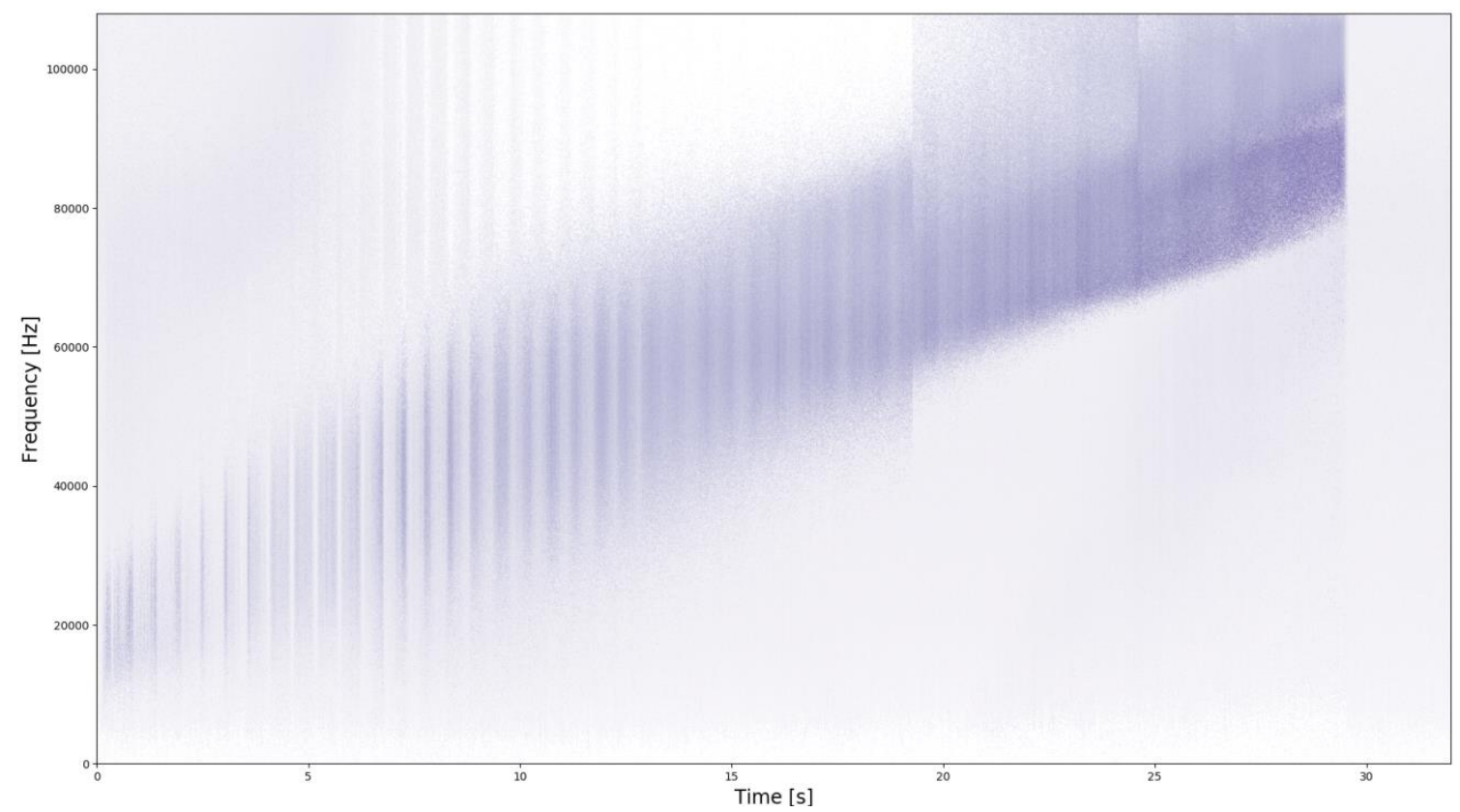

FIG. 16 Hilbert spectrum of sweep measurement between 20 and $100 \mathrm{kHz}$ with a separating wall and a hole size of $6 \mathrm{~mm}$.
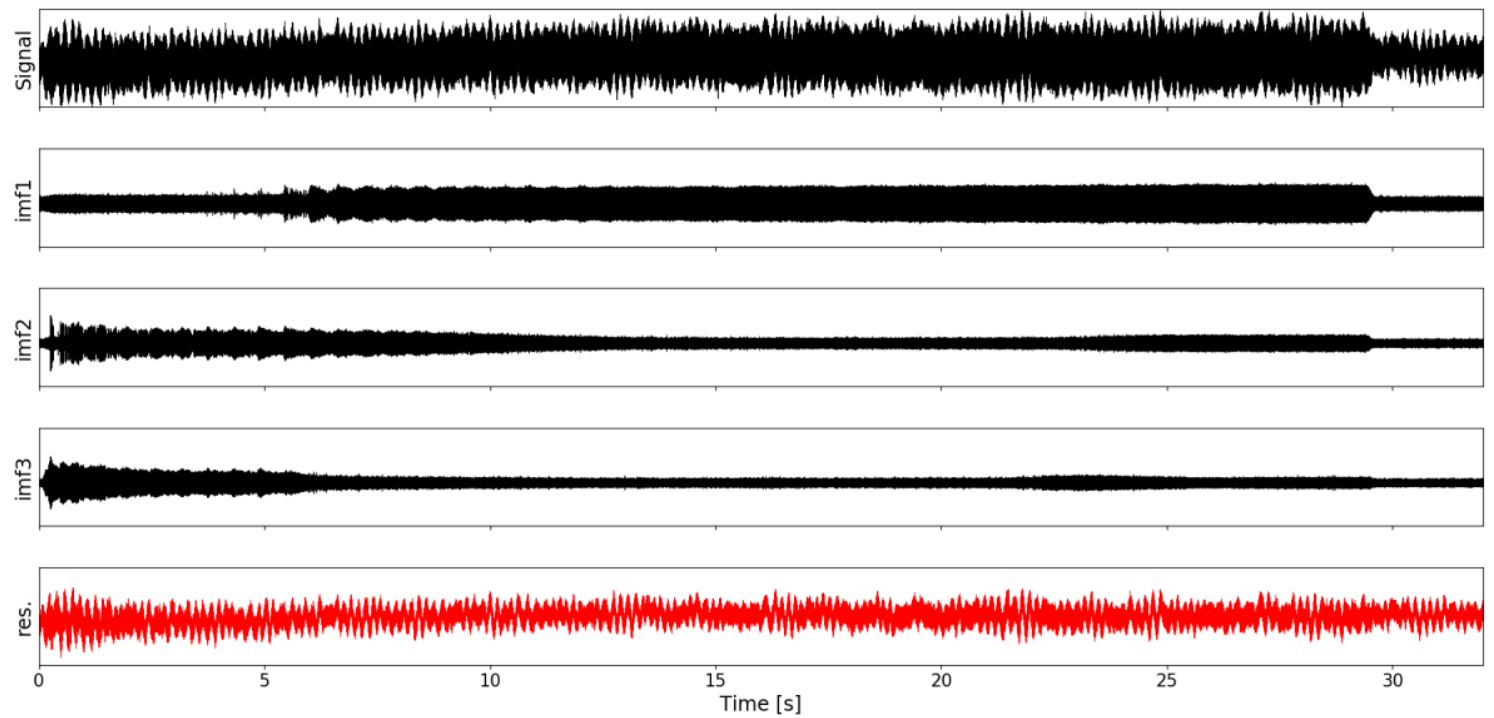

FIG. 17 EMD of the time signal into four IMFs and the remaining residual function (res.) of sweep measurement between 20 and $100 \mathrm{kHz}$ with a separating wall and a hole size of $4 \mathrm{~mm}$. 


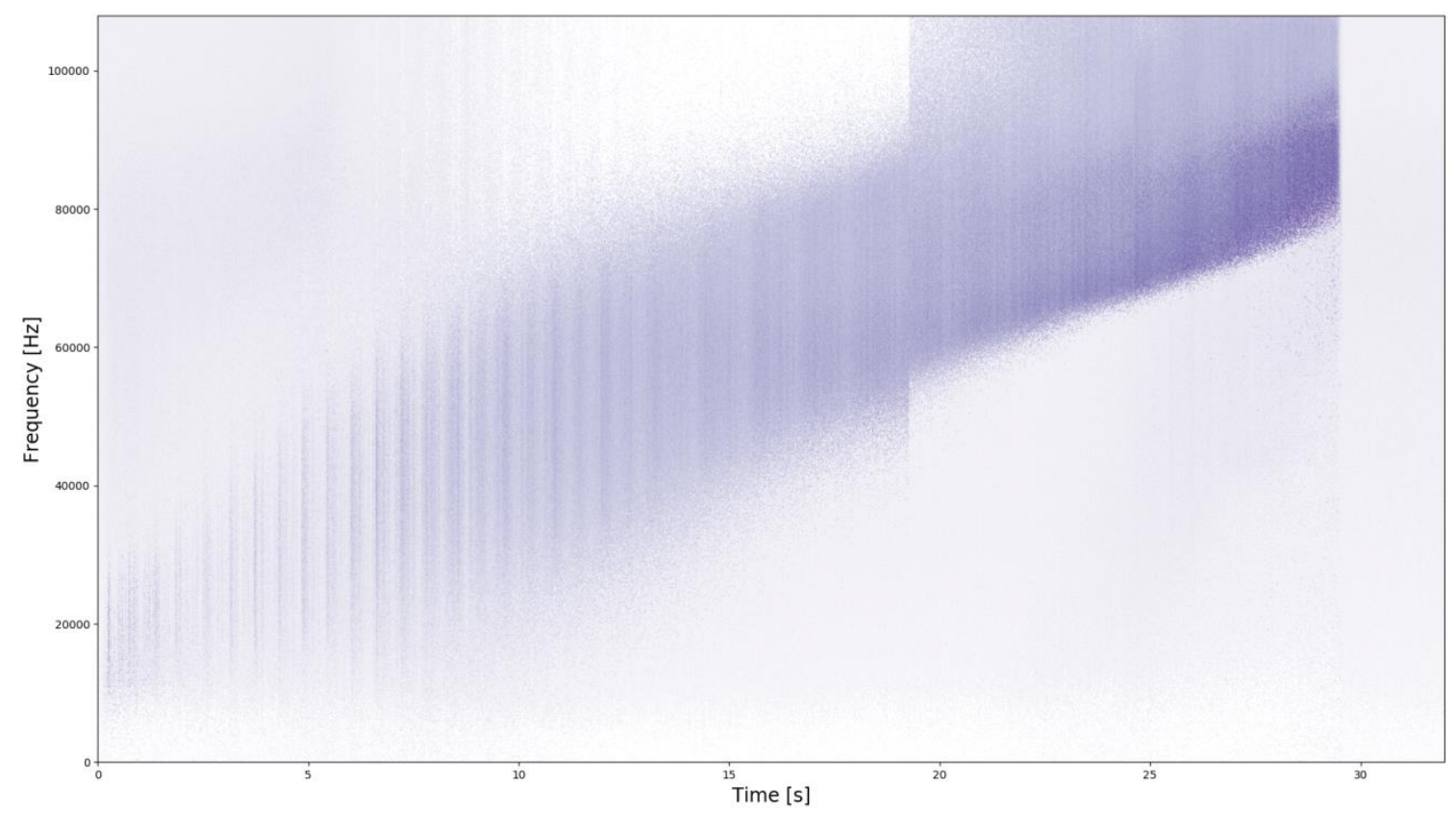

FIG. 18 Hilbert spectrum of sweep measurement between 20 and $100 \mathrm{kHz}$ with a separating wall and a hole size of $4 \mathrm{~mm}$

\section{DISCUSSION}

In figure 9, a decline of the overall frequency amplitude and stronger oscillations appears for frequencies larger than $55 \mathrm{kHz}$. The characteristics of condenser microphones changed with the wavelength of the received signal. For higher frequencies, the wavelengths became smaller and reached the order of magnitude of the microphone diaphragm at some point. In our measurements, we used a condenser microphone with a diaphragm diameter of 0.25 in. ( 6.4 mm). At this point pressure maxima and minima at the microphone diaphragm surface could cause local countermovements of the diaphragm and partial damping of the frequencies. An acoustic wavelength of $6.4 \mathrm{~mm}$ is equivalent to $54.5 \mathrm{kHz}$, and hence, to the frequency at which the decline of oscillations appeared. 
To demonstrate whether a dependency between acoustic wavelength and hole diameter existed, we tested hole sizes between $10 \mathrm{~mm}$ and $4 \mathrm{~mm}$, as shown in figure 10 . In the diameter range between $10 \mathrm{~mm}$ and $7 \mathrm{~mm}$ a correspondence between the peaks of oscillations and leakage size appeared to exist. Consequently, the comb-filtering effect may predominantly appear at larger holes, where the wavelength and the size of the hole diameter are in the same order of magnitude. For larger hole sizes, more energy was transmitted through the opening in the wall and the effect of comb filtering as more visible with increasing diameter of the holes. Under these circumstances, not only the hole size in this range may be estimated but also the distance between sound source and microphone.

Conversely, this correspondence appeared not to exist in the described test setup for hole diameters smaller than $6 \mathrm{~mm}$. As described, the acoustic wavelengths undercut the size of the microphone diaphragm at $6.4 \mathrm{~mm}$. From that point on, a correlation between the amplitude of oscillations was no longer detectable. Nonetheless, oscillations at lower frequency components still appeared and decreased with smaller leakage sizes. Therefore, the presence of a leak could be detected, but the determination of its size became more challenging for smaller leaks with this measurement setup. According to the intensity of amplification and attenuation of the frequencies, it is not yet possible to make more than an estimation of the hole size.

The calculated Hilbert spectra of hole diameters from 10 to $4 \mathrm{~mm}$ support the appearance of the comb-filtering effect in the time-frequency domain. They showed that the impact of this effect increases with increasing hole sizes. 


\section{CONCLUSION}

In this paper, we proposed a method to investigate the size of air leakage spots in building envelopes. We applied the Fourier transform and the HHT to visualize the spectral contents of recorded time signals for different leakage sizes. On the basis of these data, we observed that the leakage size could affect the appearance of the comb-filtering effect in the acoustic near field.

It has been shown that signal components pass through the openings at which frequencies corresponded to wavelengths in the range of large leakage diameters. Accordingly, the order of magnitude of leakage sizes may be deduced. For smaller diameters, the detection of leakages was still possible, but an accurate determination of the size was difficult because the dimension of the microphone diaphragm appeared to limit the detection range. In the future, the influence of different microphone dimension will be investigated to verify whether it is possible to visualize the dependency of the comb-filtering effect and leakage sizes for higher frequencies.

Using this method, leakage size detection would be independent of a blower door measurement. Airflow through the openings induced by pressure differences between inside and outside the building is not necessary for leakage size detection in this case. In contrast, other established methods, for instance, smoke tracer or infrared thermography, require enforced airflow or at least must be supported by enforced airflow.

In a next development step, it will be possible to combine this method with the application of an acoustic camera. An acoustic camera uses a microphone array and beamforming to detect the direction of sound sources. Beamforming methods in combination with the method proposed in this paper could enable the simultaneous detection of the leakage sizes as well as 
the leakage positions. Moreover, more complex hole geometries and leakage paths, as well as the extension of the frequency range to audible sound, will be examined.

\section{ACKNOWLEDGMENTS}

The present work was embedded in a joint research project of the German Aerospace Center (DLR) and the Solar-Institut Jülich at FH Aachen, which is funded by the German Ministry for Economic Affairs and Energy.

\section{REFERENCES}

1. International Energy Agency, Technology Roadmap: Energy-efficient Buildings: Heating and Cooling Equipment (Paris, France: OECD/IEA, 2011).

2. Federal Ministry of Economic Affairs and Energy, Federal Ministry of the Environment, Nature Conservation, Building and Nuclear Safety, Energiekonzept für eine umweltschonende, zuverlässige und bezahlbare Energieversorgung (Berlin, Germany: Federal Ministry of Economic Affairs and Energy, 2010).

3. Umweltbundesamt, "Berichterstattung unter der Klimarahmenkonvention der Vereinten Nationen und dem Kyoto-Protokoll 2017: Nationaler Inventarbericht zum Deutschen Treibhausgasinventar 1990-2015" (Dessau-Roßlau, 2017).

4. W. R. Chan, J. Joh, and M. H. Sherman, "Analysis of Air Leakage Measurements of US Houses," Energy and Buildings 66 (2013): 616-25, doi:10.1016/j.enbuild.2013.07.047.

5. H. Hens, Building Physics Heat, Air and Moisture: Fundamentals and Engineering Methods with Examples and Exercises (Berlin, Germany: Wilhelm Ernst \& Sohn, 2012). 
6. Standard Test Method for Determining Air Change in a Single Zone by Means of a Tracer Gas Dilution, ASTM E741-11(2017) (West Conshohocken, PA: ASTM International, 2017). www.astm.org.

7. Standard Test Method for Determining Air Leakage Rate by Fan Pressurization, ASTM E779-19 (West Conshohocken, PA: ASTM International, PA, 2019), www.astm.org

8. D. N. Keast and H.-S. Pei, "The Use of Sound to Locate Infiltration Openings in Buildings" Proceedings of the ASHRAE-DOE Conference on the Thermal Performance of the Exterior Envelope of Buildings (Orlando, FL: American Society of Heating, Refrigerating and Air Conditioning Engineers, 1979), 85-92

9. Standard Practices for Air Leakage Site Detection in Building Envelopes and Air Barrier Systems, ASTM E1186-17 (West Conshohocken, PA: ASTM International, 2017), www.astm.org

10. G. Raman, M. Prakash, R. C. Ramachandran, H. Patel, and K. Chelliah, "Remote Detection of Building Air Infiltration Using a Compact Microphone Array and Advanced Beamforming Methods" in Proceedings of Fifth Berlin Beamforming Conference ( Berlin, Germany: Gesellschaft zur Förderung angewandter Infromatik, 2014), CD-ROM

11. N. Bader, P. Holstein, K. Eckert, H.-J. Münch, L. Holtkamp, and T. van Eschut, "Akustisches Verfahren zur Dichtheitsprüfung" in Denkmal und Energie 2018: ed. B. Weller and S. Horn, (Wiesbaden, Germany, Springer Vieweg, 2017): 285-295, http://doi:10.1007/978-3-658-19672-1_22

12. A. Tiddens, J. Estevam Schmiedt, and M. Röger, System and Method for Determining the Air-Tightness of a Building. DE Patent WO 2018/104129 A1, filed November 29, 2017, and issued June 14, 2018. 
13. J. Estevam Schmiedt, D. Cerra, D. Dahlke, S. Dill, N. Ge, J. Göttsche, A. Haas, U. Heiden, M. Israel, and F. Kurz, F., "Remote Sensing Techniques for Building Models and Energy Performance Studies of Buildings," (paper presentation, EBC Annex 71: Building Energy Performance Assessment Based on In-Situ Measurements Loughborough, UK, April 26-28, 2017).

14. "Thermal Performance of Buildings - Determination of Air Permeability of Buildings Fan Pressurization Method," DIN EN ISO 9972 (Berlin: DIN Deutsches Institut für Normung e. V., 2015), https://www.din.de

15. I. S. Walker, D. J. Wilson, and M. H. Sherman, "A Comparison of the Power Law to Quadratic Formulations for Air Infiltration Calculations," Energy and Buildings 27, no. 3 (1998): 293-299, http://doi:10.1016/S0378-7788(97)00047-9

16. American Society of Heating, Refrigerating and Air- Conditioning Engineers, ASHRAE Handbook: Fundamentals-SI Edition (Atlanta, GA: American Society of Heating, Refrigerating Air-Conditioning Engineers, 2005).

17. M. H. Sherman and W. R. Chan, "Building Air Tightness: Research and Practice," Building Ventilation: The State of the Art, ed. M. Santamouris and P. Wouters (London: Earth-scan, 2006): 137-162.

18. "Acoustics-Application of New Measurement Methods in Building and Room Acoustics," DIN EN ISO 18233-SS (Berlin: DIN Deutsches Institut für Normung e. V., 2006), https://www.din.de.

19. S. Weinzierl, Handbuch der Audiotechnik (Berlin, Germany: Springer-Verlag, 2008).

20. D. F. Aldridge, "Mathematics of Linear Sweeps," Canadian Journal of Exploration Geophysics 28, no. 1 (1992): 62-68. 
21. W. Schenk, F. Kremer, G. Beddies, T. Franke, P. Galvosas, and P. Rieger, "FourierTransformation und Signalanalyse," in Physikalisches Praktikum, ed. W Schenk and F. Kremer, (Wiesbaden, Germany: Springer Fachmedien, 2014), 338-355.

22. F. A. Everest, Master Handbook of Acoustics (Blue Ridge Summit, PA: McGraw-Hill, 2001).

23. T. M. Dantas, R. P. B. Costa-Felix, and J. C. Machado, "Nonlinear Frequency Modulated Excitation Signal and Modified Compressing Filter for Improved Range Resolution and Side Lobe Level of Ultrasound Echoes," Applied Acoustics 130 (2018): 238-246, http://doi:10.1016/j.apacoust.2017.10.008

24. P. Bloomfield, Fourier Analysis of Time Series: An Introduction (New York: John Wiley, 2004).

25. J. L. Zhou and F. Wang, "Chirp Signal Time-Frequency analysis Characteristic Comparison," Applied Mechanics and Materials 226-228 (2012): 568-571.

26. N. E. Huang and S. P. Shen Samual, eds., Hilbert-Huang Transform and Its Applications, Vol. 5: Interdisciplinary Mathematical Sciences (Singapore: World Scientific, 2005), http://doi:10.1142/5862

27. N. E. Huang, Z. Shen, S. R. Long, M. C. Wu, H. H. Shih, Q. Zheng, N.-C. Yen, C. C. Tung, and H. H. Liu, "The Empirical Mode Decomposition and the Hilbert Spectrum for Nonlinear and Non-stationary Time Series Analysis," Proceedings: Mathematical, Physical and Engineering Sciences 454, no. 1971 (1998): 903-995.

28. G. Rilling, P. Flandrin, and P. Goncalves, "On Empirical Mode Decomposition and Its Algorithms" (paper presentation, IEEE-EURASIP Workshop on Nonlinear Signal and Image Processing Grado, Grado, Italy, June 8-11, 2003). 
29. N. E. Huang, Hilbert-Huang Transform and Its Applications (Singapore: World Scientific, 2014).

30. Matlab R2017b (Natick, MA, MathWorks, 2018).

31. F. J. Harris, "On the Use of Windows for Harmonic Analysis with the Discrete Fourier Transform," Proceedings of the IEEE 66, no. 1 (1978): 51-83, http://doi:10.1109/PROC.1978.10837

32. L. Bergmann, Der Ultraschall (Zurich: Hirzel Verlag, 1949). 